\title{
Comparative quality assessment of esophageal examination with transnasal and sedated endoscopy
}

\section{(ㄷ)(1) $(9)$}

\section{Authors}

Nicholas R. Crews ${ }^{1}$, Emmanuel C. Gorospe ${ }^{2}$, Michele L. Johnson², Louis-Michel Wong Kee Song ${ }^{2}$, David A. Katzka², Prasad G. Iyer ${ }^{2}$

Institutions

1 Division of Internal Medicine, Mayo Clinic, Rochester, MN, USA

2 Division of Gastroenterology and Hepatology, Mayo Clinic, Rochester, MN, USA

submitted 6.6.2016

accepted after revision $\quad 2.11 .2016$

\author{
Bibliography \\ DOI https://doi.org/10.1055/s-0042-122008 | \\ Endoscopy International Open 2017; 05: E340-E344 \\ (C) Georg Thieme Verlag KG Stuttgart · New York \\ ISSN 2364-3722
}

\section{Corresponding author}

Prasad G. Iyer, MD MS, Barrett's Esophagus Unit, Division of Gastroenterology \& Hepatology, Mayo Clinic, 200 1st St

SW, Rochester, MN, USA

Fax: +1-507-255-7652

iyer.prasad@mayo.edu

\section{ABSTRACT}

Background Unsedated transnasal esophagoscopy (TNE) may offer a less expensive, mobile alternative to sedated esophagogastroduodenoscopy (EGD) for evaluations of reflux related complications. Comparisons of imaging quality by these methods are lacking.

Methods Two reviewers evaluated videos of TNE and EGD procedures, performed during a community randomized study comparing endoscopic techniques. Subjects were randomized to EGD, TNE in endoscopy suite, or TNE in mobile research unit. Endoscopic quality was assessed using a validated scoring tool.

Results In total, 115 videos (58 EGD, 28 endoscopy suite TNE, and 29 mobile TNE) were reviewed. Overall quality scores for TNE and EGD were excellent without a statistically significant difference $(P=0.30)$. There were no differences in gastroesophageal junction (GEJ) visualization scores, though EGD scored higher in esophageal passage $(P<0.05)$ and TNE scored higher in esophageal intubation $(P<0.05)$. There was no significant difference in any quality score between mobile TNE and gastrointestinal suite TNE.

Conclusion Esophageal assessment with TNE or EGD was comparable in overall quality and GEJ visualization. TNE quality was not affected by procedure location. TNE is a feasible option for endoscopic assessment of reflux complications.

\section{Introduction}

Conventional esophagogastroduodenoscopy (EGD) is limited by the requirement for sedation and its associated recovery time, endoscopy suite resources, and anesthesia monitoring. These limitations likely decrease the effectiveness of current screening strategies for esophageal precancerous lesions [1]. Unsedated small-caliber transnasal endoscopy (TNE) has been investigated as a less expensive, efficient, office-based alternative to EGD for screening for gastroesophageal reflux complications.

TNE is a well tolerated procedure with an excellent safety profile [2-5]. The acceptability rate of TNE was $85.2 \%$ in a recent meta-analysis including 1597 patients; furthermore, 63 $80 \%$ of individuals preferred TNE to EGD for future procedures
[6-8]. Technical success rates of TNE and EGD were comparable (particularly when TNE scope diameter was $<5.9 \mathrm{~mm}$ ) [8]. TNE has also been shown to be sensitive for detecting Barrett's Esophagus (BE) in subjects with known or highly suspected BE [9-11]. Yet, the perceived TNE drawbacks including relatively inferior image quality and smaller biopsy samples have limited the integration of TNE into clinical practice for screening. Comparisons of EGD and TNE imaging quality are lacking in the community-based setting. The impact of procedural location on endoscopic imaging quality has also not been reported.

We recently conducted a randomized community trial showing that TNE screening (in the hospital endoscopy suite or mobile research van) had similar participation rates and screening yield compared to EGD [7]. In the current study, we aimed to compare the endoscopic examination quality of TNE (per- 
- Table 1 Baseline characteristics of the reviewed procedures compared to the total study procedures.

\begin{tabular}{|c|c|c|c|c|}
\hline & Total $(n=205)$ & Reviewed $(n=115)$ & Not reviewed $(n=90)$ & $P$ value \\
\hline Age, mean (SD), years & $65.5(9.2)$ & $66.5(9.2)$ & $64.3(9.0)$ & 0.08 \\
\hline Male sex, n (\%) & $94(46 \%)$ & $48(42 \%)$ & $46(51 \%)$ & 0.18 \\
\hline $\begin{array}{l}\text { Duration of endoscopic exam, mean (SD), min } \\
\text { EGD } \\
\text { hTNE } \\
\text { mTNE }\end{array}$ & $\begin{array}{l}9.3(1.6) \\
8.0(2.7) \\
8.5(2.5)\end{array}$ & $\begin{array}{l}9.3(1.7) \\
8.5(3.0) \\
8.8(2.4)\end{array}$ & $\begin{array}{l}9.1(1.0) \\
7.7(2.6) \\
8.3(2.7)\end{array}$ & $\begin{array}{l}0.68 \\
0.34 \\
0.59\end{array}$ \\
\hline Presence of esophagitis or metaplasia, n (\%) & $68(33 \%)$ & $38(33 \%)$ & $30(33 \%)$ & 0.96 \\
\hline
\end{tabular}

formed in two different settings) with EGD from this recent trial and assessed TNE imaging quality based on procedural setting.

\section{Methods}

In our recent randomized trial reported by Sami et al., 209 community subjects, 50 years or older in age without history of prior endoscopy, stratified by age, sex, and gastroesophageal reflux disease (GERD) symptoms, were recruited and randomly assigned to receive endoscopic screening by one of the three methods: TNE performed in a mobile research van unit (mTNE), TNE in a hospital endoscopy suite (hTNE), or EGD [7]. Each endoscopy was conducted by an endoscopist with TNE and EGD expertise and who had performed over 1000 upper gastrointestinal endoscopic examinations (PGI and LMW). EGD procedures were performed using a conventional high definition endoscope (GIF-180, Olympus America, Center Valley, PA, United States) under conscious sedation. hTNE and mTNE were performed using the EndoSheath ${ }^{\circledR}$ transnasal esophagoscope (TNE-5000, Vision Sciences, Orangeburg, NY, United States) following administration of nasal and oral topical anesthesia. All procedures were video recorded.

Two blinded reviewers evaluated the de-identified videos of TNE and EGD procedures. The reviewers planned to evaluate 120 videos of the 205 procedures in the trial. Videos were randomly selected proportionally (2EGD, 1 hTNE, $1 \mathrm{mTNE}$ ) from the collection to mitigate the introduction of selection bias. Procedure recording was started after oral or nasal intubation to further blind the reviewers from distinguishing TNE from EGD procedure videos. Five videos were not scored because the video was incomplete due to technical difficulties or the recording began before/during oral or nasal intubation. The reviewers were trained how to use the scoring tool and shown standardized scored videos as scoring models. Scorers were instructed to watch the video in entirety, pause the video each time before scoring, and rewind as many times as needed to ensure accurate scoring.

The reviewers assessed endoscopic quality utilizing a validated scoring tool, which was developed to evaluate the endoscopic skills of a diverse group of proceduralists (including gastroenterologists, non-gastrointestinal physicians, and physician extenders) through video review [12]. The following proficiencies were assessed: achievement of proper technique with esophageal intubation (including degree of visualization of pharyngeal and upper airway structures), achievement of proper technique with esophageal tubular passage (including maneuvering with direct vision, visualization of anatomic landmarks, proper insufflation and suctioning), percent area of gastroesophageal junction (GEJ) visualized on insertion and on withdrawal, and overall examination quality [12]. Additional details are provided in the example scoring instrument (Appendix 1). Assessment scores were recorded using a Likert scale of 1 to 5 ( 5 being the best) based on the criteria achieved in each of the five aforementioned proficiencies.

Demographic characteristics were reported using the mean and standard deviation (SD) for continuous data and the frequency and proportion for categorical data. Quality characteristics were compared using the Student's t test or chi-squared test as appropriate. Inter-rater agreement of endoscopy quality was assessed with Cohen's kappa coefficient analysis. Standard statistical software (SAS ${ }^{\circ}$ version 9.3, SAS Institute, Cary, NC, United States) was used for analysis.

\section{Results}

The reviewers scored 115 videos (58EGD, 28hTNE, and 29 mTNE). Baseline characteristics for the reviewed videos compared to the study cohort are included in $>$ Table 1 . There was no statistically significant difference in demographics, procedure time, or proportion of diagnosed esophagitis or BE in the subset of videos reviewed compared to the videos not reviewed (data not shown). The study cohort was $46 \%$ male with a mean (SD) age of 65 (9) years. Esophagitis was discovered in $32 \%$ of those screened ( 62 total, 29 Los Angeles (LA) grade A, 29 grade B, 4 grade C), while $7.8 \%$ of screened subjects had confirmed BE. Mean (SD) length of the endoscopic exam was 9.3 (1.6) minutes for EGD, 8.0 (2.7) minutes for hTNE, and 8.5 (2.5) minutes for mTNE $(P=0.51)$. Representative images taken during EGD and TNE examinations are shown in > Fig. 1 .

- Table 2 presents the quality assessment scores of the three groups. In comparisons of the EGD and combined TNE (mTNE and hTNE) groups, EGD received higher scores in tubular esophageal passage $(P<0.05)$ with a reduced number of red outs and an increased percentage of maneuvering under direct vision. The TNE group scored higher in esophageal intubation compared to EGD $(P<0.05)$ due to more frequent visualization 


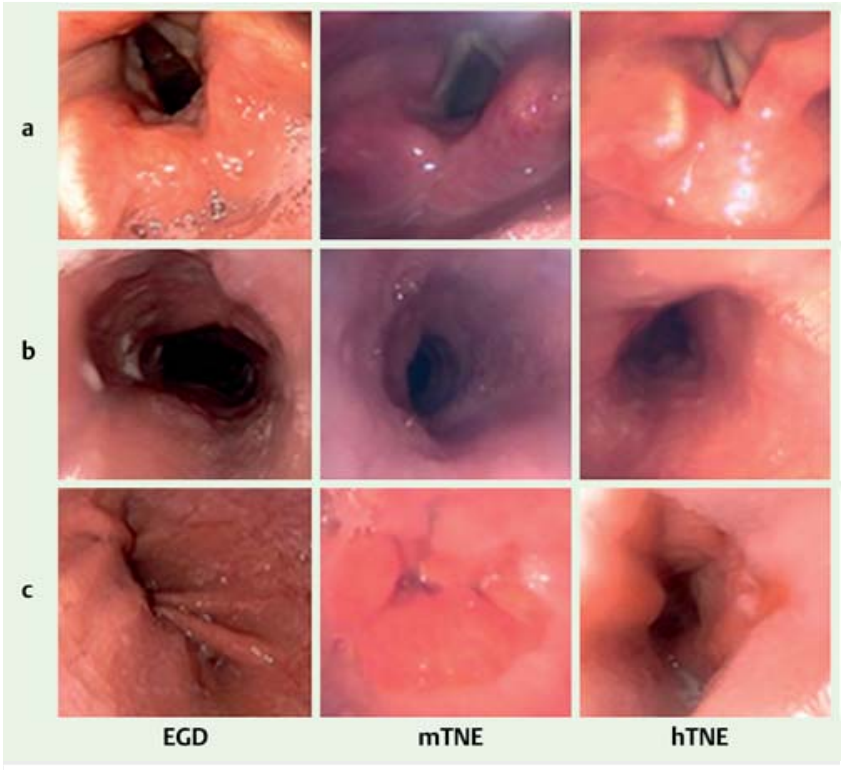

- Fig. 1 These video still-images are prototypical images of the three endoscopic methods. a shows laryngeal structures visualized. b demonstrates the tubular esophagus. c visualizes the gastroesophageal junction.

of key laryngeal anatomic structures and direct visualization of esophageal intubation posterior to the larynx. There were no differences in GEJ visualization scores with insertion $(P=0.58)$ or withdrawal $(P=0.47)$ between the groups as $>95 \%$ of the $G E J$ was visualized in nearly all cases. The overall quality scores for TNE and EGD were excellent without statistically significant differences $(P=0.30)$. There was no significant difference in any quality assessment score between mTNE and hTNE groups, which were different only in procedural location. Inter-rater agreement of endoscopy quality was excellent (kappa $=0.88)$.

\section{Discussion}

In this brief report, we found that the quality of esophageal assessment with TNE (conducted in a gastrointestinal endoscopy suite (hTNE) or in a mobile research van (mTNE)) was comparable to EGD in terms of overall quality and GEJ visualization. TNE received higher esophageal intubation scores, likely due to passage through the nasal cavity into the superior oropharynx, which provided superior visualization of the laryngeal and oropharyngeal structures. EGD scored higher in tubular esophagus passage metrics likely due to greater insufflation capabilities compared to the ultrathin TNE endoscope. TNE quality was not affected by procedure setting as there was no difference in quality scores between the mTNE and hTNE groups. These results indicate that TNE using the EndoSheath technology is a feasible efficient option for endoscopic assessment of reflux complications in a population-based setting.

The scoring tool employed in this study to assess endoscopic quality was validated in a large multicenter study performed in the UK that included over 4000 subjects with a diverse group of endoscopists [12]. That study had some limitations. The scoring tool did not include assessments to evaluate the endoscopist's diagnostic yield or esophageal lesion identification. In our previously published study, Sami et al. reported no difference in suspected or confirmed BE rates between the three arms (EGD, mTNE, or hTNE) of the study [7]. These results are akin to multiple studies showing that TNE is comparable to EGD at detecting BE [9-11]. The scoring tool also did not assess the endoscopist's level of comfort or perceived difficulty of the procedure. These additional personal viewpoints could be incorporated into future investigations of TNE used in primary care settings with endoscopists less experienced with TNE. It was also difficult to completely blind reviewers from the potentially different appearances of TNE and EGD videos. Nevertheless, by applying a clear scoring system, it was hoped that videos were scored on their merit rather than as a comparison.

As we have previously reported, there was no difference in the rate of successful intubation, complete evaluation, or BE

- Table 2 Comparison between TNE and EGD quality assessment scores.

\begin{tabular}{|c|c|c|c|c|c|}
\hline Quality assessment score & $\begin{array}{l}\text { Group EGD } \\
(n=58)\end{array}$ & $\begin{array}{l}\text { Group mTNE } \\
(n=29)\end{array}$ & $\begin{array}{l}\text { Group hTNE } \\
(n=28)\end{array}$ & $\begin{array}{l}P \text { value for comparison } \\
\text { of EGD vs. all TNE }\end{array}$ & $\begin{array}{l}P \text { value for comparison } \\
\text { of mTNE vs. hTNE }\end{array}$ \\
\hline $\begin{array}{l}\text { Esophageal intubation score, } \\
\text { mean (SD) }\end{array}$ & $1.5(1.6)$ & $3.8(1.1)$ & $3.3(1.9)$ & $<0.05^{1}$ & 0.21 \\
\hline $\begin{array}{l}\text { Tubular esophagus passage } \\
\text { score, mean (SD) }\end{array}$ & $3.6(0.7)$ & $2.3(0.7)$ & $2.5(1.1)$ & $<0.05^{1}$ & 0.34 \\
\hline $\begin{array}{l}\text { GEJ visualization score during } \\
\text { insertion, mean (SD) }\end{array}$ & $4.9(0.2)$ & $4.9(0.3)$ & $4.9(0.2)$ & 0.58 & 0.59 \\
\hline $\begin{array}{l}\text { GEJ visualization score during } \\
\text { withdrawal, mean (SD) }\end{array}$ & $4.9(0.3)$ & $4.9(0.3)$ & $4.8(0.4)$ & 0.47 & 0.53 \\
\hline $\begin{array}{l}\text { Overall esophageal examination } \\
\text { score, mean (SD) }\end{array}$ & $4.1(0.2)$ & $4.1(0.3)$ & $4.0(0.1)$ & 0.42 & 0.22 \\
\hline
\end{tabular}


screening yield between the three arms (EGD, mTNE, or hTNE) of this prospective population-based study [7]. Comparing TNE and EGD examinations, the absolute difference in the mean procedure length was minimal in this study, likely due to the research protocol driven acquisition of GEJ and esophageal biopsies in all three arms. However, recovery time (extubation to discharge) was substantially longer by approximately 50 minutes for subjects who received sedated EGD compared to TNE [7]. This decreased recovery time associated with TNE is beneficial for the patient as well as the endoscopist. By including a disposable sheath, TNE also avoids the need for conventional endoscope sterilization between procedures. Given that TNE examination quality did not differ by procedure setting, TNE could likely be employed effectively in an office-based setting, community center or even a mobile research unit as investigated in this study for assessment of complications from gastroesophageal reflux. Furthermore, a majority of primary care providers may be more willing to refer patients for screening with TNE if it was readily available (62\%) and to perform TNE in their office if trained (52\%) [13]. These time-saving, mobile features associated with TNE are ideal for increasing case volume and accessibility which would be necessary in community-based BE screening programs.

Current limitations for TNE include the lack of readily available TNE training opportunities and current endoscopists with TNE experience. However, recent studies suggest that these shortages are not insurmountable. Alashkar et al. recently reported that physician assistants and nurse practitioners can be trained to conduct BE screening with TNE and gain proficiency in 50 procedures [14]. Reasonable, plausible TNE training strategies using available providers and resources need to be further delineated.

In conclusion, the overall quality of esophageal assessment and GEJ visualization was comparable for TNE performed using the EndoSheath technology and EGD in a community cohort. TNE quality was not affected by procedure location and thus could be used effectively in mobile or office-based settings. These results indicate that TNE is a feasible option for endoscopic assessment of reflux complications, and can be used in a community setting.

\section{Acknowledgments}

Funded by NIH grant (RC4DK090413). This publication was made possible by CTSA Grant Number UL1 TR000135, from the National Center for Advancing Translational Sciences (NCATS), a component of the National Institutes of Health (NIH). Its contents are solely the responsibility of the authors and do not necessarily represent the official view of NIH.
Competing interests

None

References

[1] Iyer PG, Chak A. Can endosheath technology open primary care doors to Barrett's esophagus screening by transnasal endoscopy? Endoscopy 2016; 48: 105-106

[2] Peery AF, Hoppo T, Garman KS et al. Feasibility, safety, acceptability, and yield of office-based, screening transnasal esophagoscopy (with video). Gastrointest Endosc 2012; 75: 945 - 953, e942

[3] Chung JW, Park S, Chung M] et al. A novel disposable, transnasal esophagoscope: a pilot trial of feasibility, safety, and tolerance. Endoscopy 2012; 44: $206-209$

[4] Mokhashi MS, Wildi SM, Glenn TF et al. A prospective, blinded study of diagnostic esophagoscopy with a superthin, stand-alone, batterypowered esophagoscope. Am J Gastroenterol 2003; 98: 2383-2389

[5] Garcia RT, Cello JP, Nguyen MH et al. Unsedated ultrathin EGD is well accepted when compared with conventional sedated EGD: a multicenter randomized trial. Gastroenterology 2003; 125: 1606 - 1612

[6] Jobe BA, Hunter JG, Chang EY et al. Office-based unsedated small-caliber endoscopy is equivalent to conventional sedated endoscopy in screening and surveillance for Barrett's esophagus: a randomized and blinded comparison. Am J Gastroenterol 2006; 101: 2693-2703

[7] Sami SS, Dunagan KT, Johnson ML et al. A randomized comparative effectiveness trial of novel endoscopic techniques and approaches for Barrett's esophagus screening in the community. Am J Gastroenterol 2015; 110: $148-158$

[8] Sami SS, Subramanian V, Ortiz-Fernández-Sordo J et al. Performance characteristics of unsedated ultrathin video endoscopy in the assessment of the upper gastrointestinal tract: systematic review and metaanalysis. Gastrointest Endosc 2015; 82: 782 - 792

[9] Shariff MK, Bird-Lieberman EL, O’Donovan M et al. Randomized crossover study comparing efficacy of transnasal endoscopy with that of standard endoscopy to detect Barrett's esophagus. Gastrointest Endosc 2012; 75: $954-961$

[10] Saeian K, Staff DM, Vasilopoulos S et al. Unsedated transnasal endoscopy accurately detects Barrett's metaplasia and dysplasia. Gastrointest Endosc 2002; 56: 472-478

[11] Aedo MR, Zavala-Gonzalez MA, Meixueiro-Daza A et al. Accuracy of transnasal endoscopy with a disposable esophagoscope compared to conventional endoscopy. World J Gastrointest Endosc 2014; 6: 128 136

[12] Williams ], Russell I, Durai D et al. What are the clinical outcome and cost-effectiveness of endoscopy undertaken by nurses when compared with doctors? A Multi-Institution Nurse Endoscopy Trial (MINuET) Health Technol Assess 2006; 10: 1-195 iii-iv, ix-x

[13] Boolchand V, Faulx A, Das A et al. Primary care physician attitudes toward endoscopic screening for GERD symptoms and unsedated esophagoscopy. Gastrointest Endosc 2006; 63: 228-233

[14] Alashkar B, Faulx AL, Hepner A et al. Development of a program to train physician extenders to perform transnasal esophagoscopy and screen for Barrett's esophagus. Clin Gastroenterol Hepatol 2014; 12 : $785-792$ 


\section{Appendix 1 - UPPER ENDOSCOPY EVALUATION SHEET}

\begin{tabular}{|l|l|l|}
\hline Video ID: & Scorer: & Date: \\
\hline
\end{tabular}

Time, you began scoring: $\square$ hr $\square$ min $\square$ sec

\section{Section A - Dexterity and Safety}

This section is to evaluate dexterity and safety of performance of OGD. This includes instrument entry, passage, and manipulation through the mouth, throat, and oesophagus. Please check various items under each item before you score. Pause the video each time before scoring and rewind as many times as needed to ensure accurate scoring.

\section{Oesophageal intubation}

$\square$ Passage under direct vision all the time

$\square$ Following centre of tongue

$\square$ Visualizing epiglottis

$\square$ Visualize the cricoarytenoid folds and vocal cords

$\square$ Insertion posterior to the larynx between the pyriform sinuses

\section{Passage through oesophagus}

$\square$ Insertion under direct vision all the time

$\square$ No mucosal red or white outs

$\square$ Adequate air insufflation

$\square$ No mucosal wall collisions

$\square$ Suctioning any secretions

3. Examination of OG junction (Z-line or squamocolumnar junction in particular)

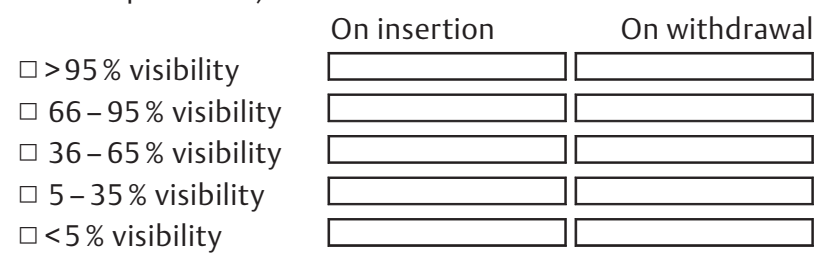

\section{Overall Score of Oesophageal Exam}

$\square$ Complete examination and no concerns over technique or content

$\square$ Probably complete examination with minor concern over technique or content

$\square$ Incomplete examination with moderate concern over technique or content

$\square$ Incomplete examination with major concern over technique or content

$\square$ Incomplete examination and totally unacceptable technique or content

Time, when you finish scoring: $\square$ hr $\square$ min $\square$ sec 